\title{
Integrated Micro-Optics for Microfluidic Detection
}

\author{
Yuto KAZAMA* and Akihide HibARA**广 \\ *Department of Applied Chemistry, School of Engineering, The University of Tokyo, 7-3-1 Hongo, Bunkyo, \\ Tokyo 113-8550, Japan \\ **Department of Chemistry, Graduate School of Science and Engineering, Tokyo Institute of Technology, \\ 2-12-1-W4-20 Ookayama, Meguro, Tokyo 152-8551, Japan
}

\begin{abstract}
A method of embedding micro-optics into a microfluidic device was proposed and demonstrated. First, the usefulness of embedded right-angle prisms was demonstrated in microscope observation. Lateral-view microscopic observation of an aqueous dye flow in a 100- $\mu \mathrm{m}$-sized microchannel was demonstrated. Then, the embedded right-angle prisms were utilized for multi-beam laser spectroscopy. Here, crossed-beam thermal lens detection of a liquid sample was applied to glucose detection.
\end{abstract}

Keywords Microfluidics, optics, polydimethylsiloxane, embedded optics, thermal lens

(Received August 28, 2015; Accepted September 30, 2015; Published January 10, 2016)

\section{Introduction}

Micro/nanofabrication technologies have been important in the field of miniaturized total analytical systems ( $\mu$ TAS) and lab-on-chip. ${ }^{1-3}$ These technologies contribute to reducing the necessary amount of reagents, analytical time, and size of apparatus. In miniaturized chemical/biochemical analysis, soft lithography technology utilizing polydimethylsiloxane (PDMS) is a powerful tool because of its simplicity. ${ }^{4-7}$ Since PDMS is solidified from a precursor liquid, it can not only be used to construct a microstructure from a mold but also for embedding small functional objects, such as permeable membranes, into the microdevice. ${ }^{8-11}$ By utilizing this capability, various conventional technologies may be integrated into micro analytical devices.

Spectroscopic and/or microscopic tools are crucially important for micro analytical devices. ${ }^{6}$ With regard to optical detection tools, the integration methods of light emission diodes, ${ }^{12}$ optical fibers, ${ }^{12,13}$ photodiodes, ${ }^{14}$ and lithographic lenses ${ }^{15}$ were reported in the early 2000s. These technologies have been used in various micro/nanofluidic applications. For example, embedded fibers have been widely utilized in multiplex fluorescent detection $^{13}$ and optofluidics. ${ }^{16}$ These embedded optics seem very powerful for fluorescent and absorption detection, but they have not been applied to advanced laser spectroscopy methods, such as thermal lens detection, that have been widely used in micro/nanofluidic analyses. ${ }^{17,18}$ When micro-optics developed for free-space optical systems are combined with conventional technologies, the application range of the embedded optics can be expanded significantly.

In this paper, an embedding method for small-size optics is proposed and verified, and thermal lens detection on a microfluidic device is demonstrated. Thermal lens detection

$\dagger$ To whom correspondence should be addressed.

E-mail: ahibara@chem.titech.ac.jp using embedded prisms was conceived as shown in Fig. $1 .{ }^{19}$ Excitation and probe beams are introduced to the embedded prism from the normal direction, being focused by the lithographic lens, and cross in a microchannel. By utilizing the embedded prism, a lateral-view (side-view) observation of the microchannel flow is possible (right side of Fig. 1).

\section{Experimental}

\section{Chip design}

Figure 2 shows the design of a microfluidic chip. The central straight line denotes a microchannel, which has a square part for right-angle beam introduction. There are four prism guides for laser beam introduction and output. The right-angle prisms are designed to be aligned to these mold structures before PDMS solidification. The four concave structures are focusing lenses.

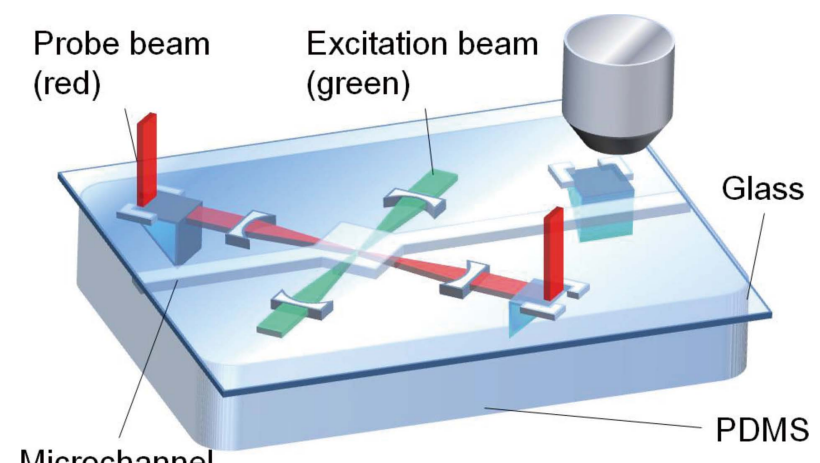

Microchannel

Fig. 1 Concept of microfluidic analytical device with embedded prisms. The figure is adopted from Ref. 19 with the permission of the Transducers Research Foundation. 


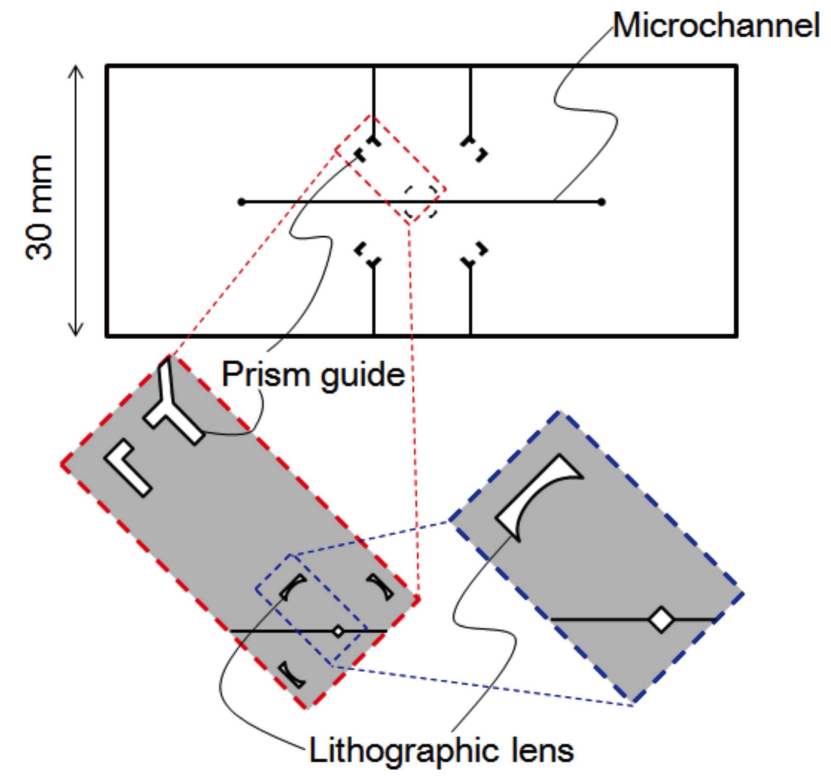

Fig. 2 Design of microfluidic device.
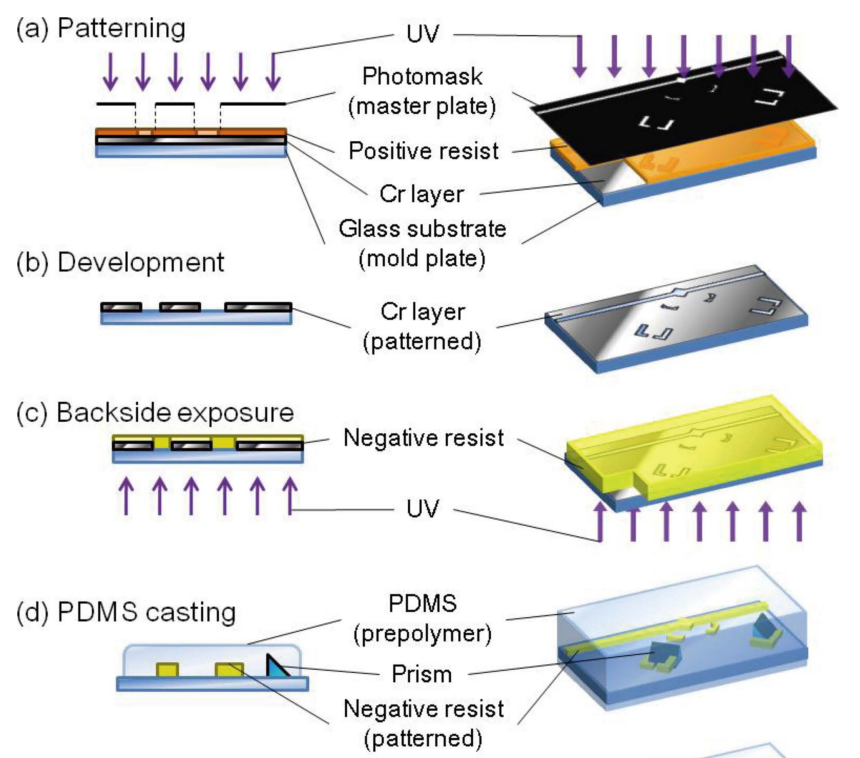

(e) Curing, peeling off, bonding
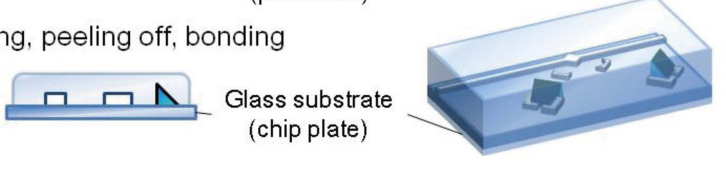

Fig. 3 Fabrication process.

After bonding PDMS to the glass, the parts are vacant and the refractive indices are lower than the surrounding PDMS $(n-1.41)$. Therefore, the concave structures act as focusing lenses.

\section{Chip fabrication}

Figure 3 is a schematic of the fabrication process, with top-view illustrations (right) presented in addition to the side view (left). In Fig. 3, only a part of the pattern in Fig. 2 is schematically illustrated. A microfluidic/optics pattern was drawn on a master photomask (Cr/glass) substrate by using an electron beam patterning. Passing through the photomask, UV
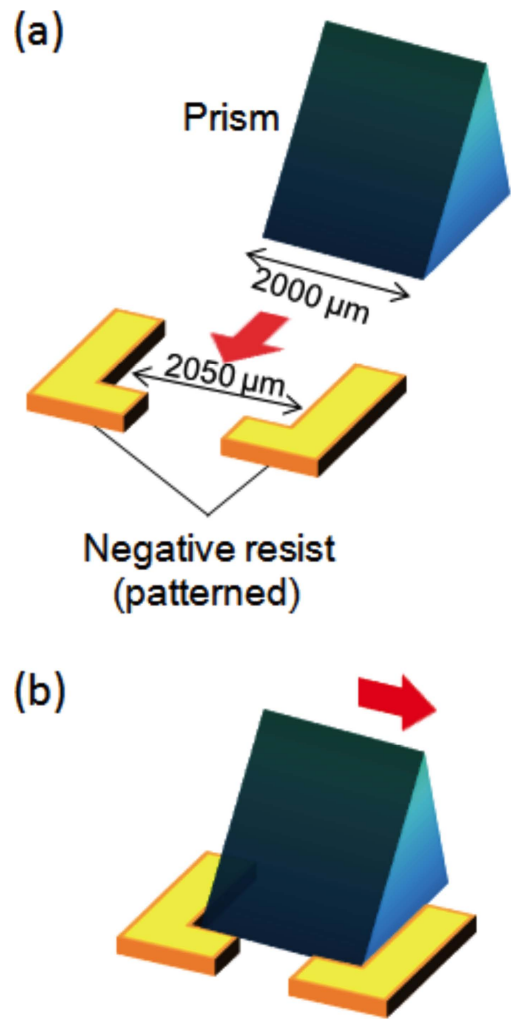

Fig. 4 Schematic illustration of prism positioning. (a) The prism was aligned first to the long side having a gap for better contact of PDMS to the prism. (b) The prism was aligned first to one of the short sides next.

light irradiated a positive photoresist layer on another Cr-coated glass substrate (mold plate) (Fig. 3(a)). After development, a mold plate with a Cr pattern was obtained (Fig. 3(b)). Then, a negative thick photoresist (KMPR-1035) layer $(80 \mu \mathrm{m})$ was spin-coated on the mold substrate ( $\mathrm{Cr}$ side). UV light irradiated the resist layer on the mold substrate from the backside (the glass side) through the on-substrate pattern (Fig. 3(c)). This operation ensures better lithography resolution (see Supporting Information A), which is especially important for the precision of the lithography lens. After development, a mold for a microchannel and setting-guide-structures for the prisms was obtained. Right-angle prisms having 2-mm square bases were placed on the mold. For precise positioning of the prisms, the setting-guide structures illustrated in Fig. 4 were fabricated. The spacing between the structures was designed to be slightly wider than the prism. The prism was aligned first to the long side having a gap for better contact of PDMS to the prism, and to one of the short sides next. This procedure gave good alignment reproducibility (see Supporting Information B). After the alignment, a prepolymer of PDMS was casted and thermally polymerized (Fig. 3(d)). The PDMS sheet was peeled off with the prisms and bonded to a glass plate (chip plate) (Fig. 3(e)).

\section{Thermal lens detection}

An excitation laser (J050GS-11 Showa Optronics, $\lambda=532 \mathrm{~nm}$, $50 \mathrm{~mW}, 80 \mathrm{~Hz}$ modulated by optical chopper) and a probe laser (LPS-660-FC Thorlabs, $\lambda=660 \mathrm{~nm}, 3 \mathrm{~mW}$ ) were introduced to the chip through the embedded prisms. The divergence of the output probe beam was detected with a photodiode (ET-2030, Electro-Optics Technology). The photodiode signal was fed 
into a lock-in amplifier (LI5640, NF Corporation), and the thermal lens signal was recorded on a computer.

\section{Materials}

A glucose assay kit (FN-EBGL100, Gentaur) was used for the thermal lens detection, where the glucose oxidase reaction produced a colored compound having a maximum absorption wavelength of $570 \mathrm{~nm}$. Right-angle prisms (2MM AL+MGF2, Edmund Optics) were used for the embedded optics. (a)
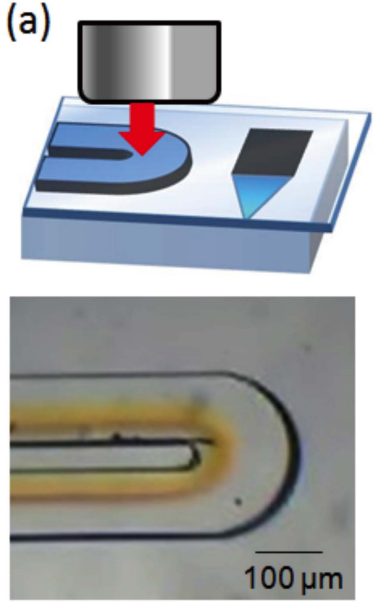

(b)

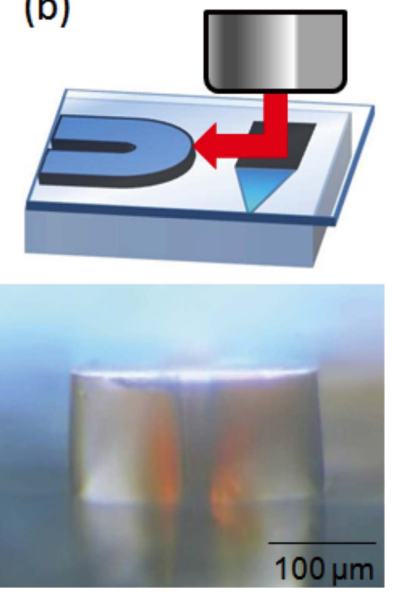

Fig. 5 (a) Top-view of microchannel flow. microchannel flow.

\section{Results and Discussion}

First, the lateral-view concept was verified. Here, a dye (Sunset Yellow) aqueous solution was introduced from a side channel of the $\psi$-shaped channel ( 3 inlets and 1 outlet), while a pure solvent (water) was introduced from the center and other side channels. As shown in Fig. 5(a), the dye flow was successfully localized to the inner part of the turn channel. When the same situation was observed from the side as shown in Fig. 5(b), the dye solution flow was successfully visualized. Figure 5(b) shows the dye flowing from left to right. While the dye was localized in the inner wall, it became slightly dim after passing the turn structure. This means a certain distortion of the flow by the turn structure was observed. Observing from the side seems very attractive for flow analysis. When particles are added to the solution, 3D visualization is possible in principle by using the particles' defocus information. The $3 \mathrm{D}$ visualization will be discussed elsewhere.

Photothermal spectroscopy is well known as a highly sensitive tool for detecting nonfluorescent compounds, ${ }^{20,21}$ where thermal lens detection is often used in microfluidic applications. ${ }^{22}$ In thermal lens detection, a non-fluorescent sample solution absorbs the excitation laser, and the absorbed light energy is released as thermal energy via non-radiative relaxation to increase temperature around the excitation beam. The temperature increase corresponds to a refractive index change. The temperature gradient around the excitation beam acts as a transient concave lens (thermal lens effect). The locus of the probe beam is modified by the transient lens, and the degree of the lens effect can be measured by the probe beam divergence. The degree of the lens effect is proportional to the released thermal energy, that is, the concentration of sample compound (a)
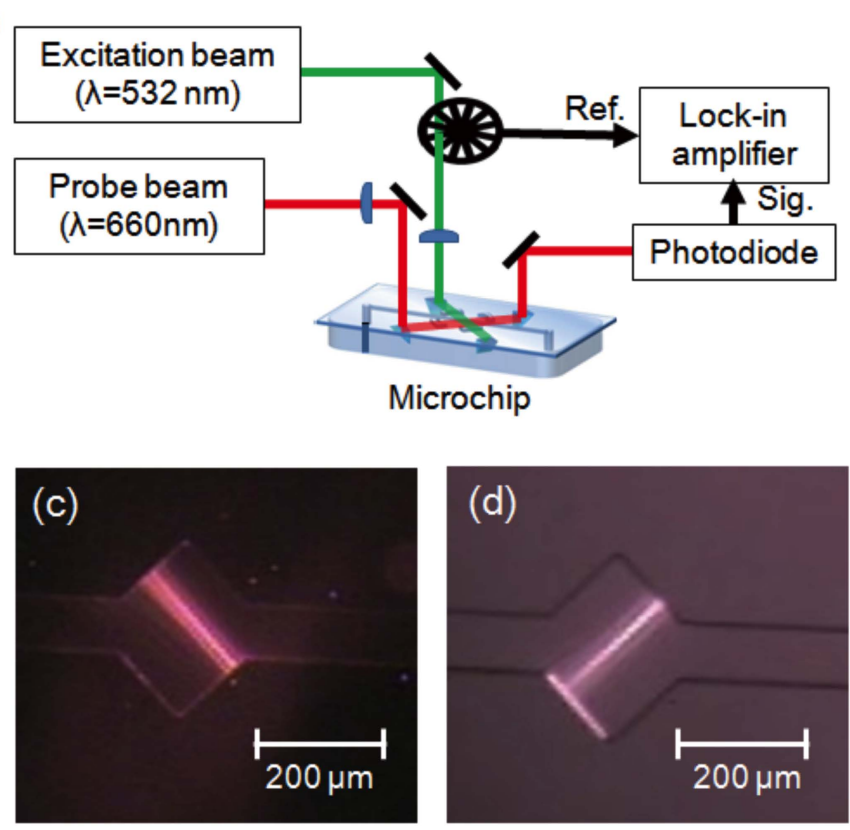

(b)
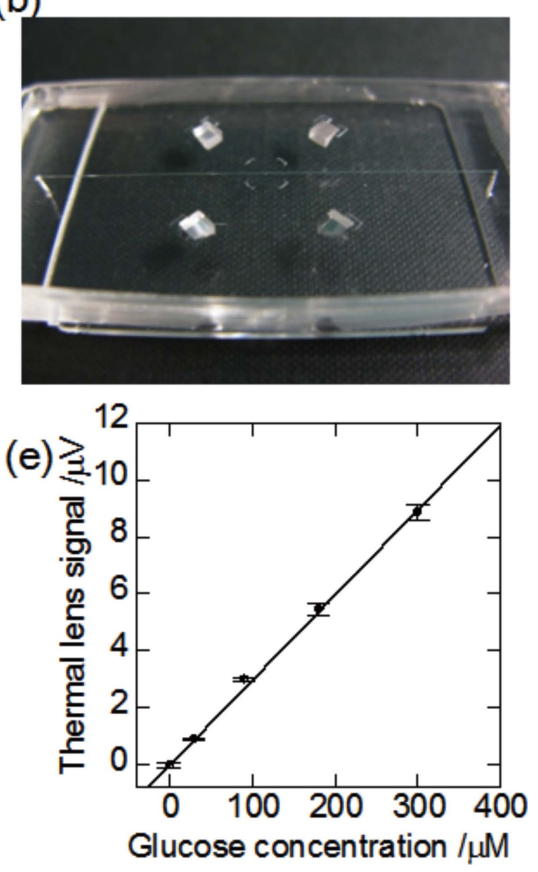

Fig. 6 (a) Diagram of thermal lens detection setup. (b) Photograph of the microchip. (c) Locus of the excitation beam. (d) Locus of the probe beam. (e) Thermal lens signal dependence on glucose concentration. The figures (c) and (d) are adopted from Ref. 19 with the permission of the Transducers Research Foundation. 
in the solution.

There are several types of thermal lens detection, such as the coaxial-beam ${ }^{17,23}$ and the crossed-beam ${ }^{24}$ configurations. In this paper, crossed-beam thermal lens detection is demonstrated. The conceptual optical configuration is shown in Fig. 1. In order to realize the conception, the optical system shown in Fig. 6(a) was set up. In order to visualize the loci of both the excitation and probe beams, an aqueous solution of red fluorescent dye was introduced into the microchannel. Figures 6(c) and 6(d) show the loci of the excitation and the probe beams, respectively. The beams were focused by the lithography lens and crossed in the microchannel as designed. Figure 6(e) shows the calibration of glucose by thermal lens detection. The calibration line was linear in the range of the assay kit $(R=$ 0.998). Although sensitivity of the present setup was not so high and there is still room for optimization, multi-beam laser spectroscopic detection was successfully demonstrated by utilizing the embedded optics.

\section{Conclusions}

An embedding method for small-size optics was proposed and verified. By utilizing embedded right-angle prisms, lateral view observation of the microchannel flow and thermal lens detection in the microchannel were demonstrated successfully. The embedding method can be used in various manners; for example, in an angle-resolved laser scattering method and in 3D microflow visualization.

\section{Acknowledgements}

A part of this study was supported by JSPS KAKENHI Grant Number 15H03825. Y. K. acknowledges the support from the Global Center of Excelence (GCOE) Program of Japan's Ministry of Education, Culture, Sports, Science adn Technology and the JSPS International Training Program.

\section{Supporting Information}

Additional data on the backside exposure and the prism alignment are shown in the Supporting Information. This material is available free of charge on the Web at http://www. jsac.or.jp/analsci/.

\section{References}

1. M. L. Kovarik, D. M. Ornoff, A. T. Melvin, N. C. Dobes, Y.
L. Wang, A. J. Dickinson, P. C. Gach, P. K. Shah, and N. L. Allbritton, Anal. Chem., 2013, 85, 451.

2. P. A. Auroux, D. Iossifidis, D. R. Reyes, and A. Manz, Anal. Chem., 2002, 74, 2637.

3. D. R. Reyes, D. Iossifidis, P. A. Auroux, and A. Manz, Anal. Chem., 2002, 74, 2623.

4. S. Masuda, M. Washizu, and T. Nanba, IEEE Trans. Ind. Appl., 1989, 25, 732.

5. R. Mukhopadhyay, Anal. Chem., 2007, 79, 3248.

6. B. Kuswandi, Nuriman, J. Huskens, and W. Verboom, Anal. Chim. Acta, 2007, 601, 141.

7. A. D. Stroock and G. M. Whitesides, Electrophoresis, 2002, 23,3461 .

8. R. F. Ismagilov, J. M. K. Ng, P. J. A. Kenis, and G. M. Whitesides, Anal. Chem., 2001, 73, 5207.

9. K. J. Lee, K. A. Fosser, and R. G. Nuzzo, Adv. Funct. Mater, 2005, 15, 557.

10. J. Kim and B. K. Gale, Lab Chip, 2008, 8, 1516.

11. Y. Imura, E. Yoshimura, and K. Sato, Anal. Sci., 2012, 28, 197.

12. K. Uchiyama, W. Xu, J. Qiu, and T. Hobo, Fresenius' J. Anal. Chem., 2001, 371, 209.

13. R. H. Cole, N. de Lange, Z. J. Gartner, and A. R. Abate, Lab Chip, 2015, 15, 2754.

14. M. L. Chabinyc, D. T. Chiu, J. C. McDonald, A. D. Stroock, J. F. Christian, A. M. Karger, and G. M. Whitesides, Anal. Chem., 2001, 73, 4491.

15. S. Camou, H. Fujita, and T. Fujii, Lab Chip, 2003, 3, 40.

16. M. Rosenauer and M. J. Vellekoop, Lab Chip, 2009, 9, 1040.

17. T. Kitamori, M. Tokeshi, A. Hibara, and K. Sato, Anal. Chem., 2004, 76, 52a.

18. M. A. Proskurnin, E. S. Ryndina, D. S. Tsar'kov, V. M. Shkinev, A. Smirnova, and A. Hibara, Anal. Sci., 2011, 27, 381.

19. Y. Kazama and A. Hibara, in The 14th International Conference on Miniaturized Systems for Chemistry and Life Sciences, 2010, Transducers Research Foundation, Groningen, The Netherlands, 1262.

20. D. A. Nedosekin, E. I. Galanzha, E. Dervishi, A. S. Biris, and V. P. Zharov, Small, 2014, 10, 135.

21. P. Vermeulen, L. Cognet, and B. Lounis, J. Microsc., 2014, $254,115$.

22. V. S. Dudko, A. P. Smirnova, M. A. Proskurnin, A. Hibara, and T. Kitamori, Russ. J. Gen. Chem., 2012, 82, 2146.

23. K. Uchiyama, A. Hibara, H. Kimura, T. Sawada, and T. Kitamori, Jpn. J. Appl. Phys. 1, 2000, 39, 5316.

24. D. S. Burgi and N. J. Dovichi, Appl. Opt., 1987, 26, 4665. 